\title{
COEXISTENCE OF PSORIASIS WITH BULLOUS PEMPHIGOID: A CASE REPORT
}

\author{
S. Dhanyasree ${ }^{1}$, Ajaykumar $^{2}$, Chenna Subhashini ${ }^{3}$, P. Anila Sunandhini ${ }^{4}$, V. Srilakshmi ${ }^{5}$
}

\section{HOW TO CITE THIS ARTICLE:}

S. Dhanyasree, Ajaykumar, Chenna Subhashini, P. Anila Sunandhini, V. Srilakshmi. "Coexistence of Psoriasis with Bullous Pemphigoid: A Case Report". Journal of Evolution of Medical and Dental Sciences 2015; Vol. 4, Issue 69, August 27; Page: 12092-12096, DOI: 10.14260/jemds/2015/1743

\begin{abstract}
Psoriasis and bullous pemphigoid (BP) represent two clinically well-characterized, chronic, inflammatory skin conditions.(1) The concomitant occurrence of these two entities in a patient is rare(2) Here we report a 65-year-old female suffering from psoriasis vulgaris for 15 years on irregular medication who noticed eruption of blisters all over the body. Direct immunefluorescence and histopathological examination confirmed the diagnosis of bullous pemphigoid. In our case, bullous eruptions were successfully treated with tetracycline and dapsone, and psoriasis with methotrexate. We want to report the case as there are very few case reports available with psoriasis coexistence with bullous pemphigoid.
\end{abstract}

KEYWORDS: Psoriasis, Bullous pemphigoid, Methotrexate, Tetracycline, Dapsone.

INTRODUCTION: Psoriasis is a chronic T-cell-mediated inflammatory disease affecting the skin and joints. Psoriasis has been consistently associated with many cutaneous and systemic conditions. Recent epidemiological studies shown that psoriasis patients are prone to develop cardiovascular and other metabolic syndromes. On the other hand, a variety of cutaneous disorders may be associated with psoriasis. Occurrence of autoimmune blistering disease in psoriasis is rare, here we report a case of coexistence of psoriasis with bullous pemphigoid (BP).(3,4)

CASE STUDY: A 65 years old female presented with history of blisters all over the body of 7 days duration. They were associated with itching. The blisters were noticed first on the arm and later spread to other parts of the body. She also gave history of oral ulcers. The patient was a known case of psoriasis vulgaris since 15 years. She had used both modern medicines and alternative medicines for psoriasis for a variable period of time. But she was on irregular treatment. Cutaneous examination was revealed tense bullae and crusted erosions on upper and lower limbs (Fig. 2, 3). Bulla spread sign and nikolsky's sign were negative. There were scaly plaques on the trunk and extremities (Fig. 1, 3).Blisters were present over the both scaly plaques as well as on the normal skin. A diagnosis of psoriasis vulgaris with BP was considered and she was investigated. Her haematological and biochemical parameters were normal.

The skin biopsy from scaly plaque was consistent with the diagnosis of psoriasis and showed hyperkeratosis, parakeratosis, acanthosis, regular elongation of rete ridges (Fig. 4, 5). Skin biopsy from the vesicular lesion showed normal epidermis with subepidermal cleft with inflammatory infiltrate at the roof and the floor of the blister, predominantly eosinophils. $(5,6)$ Direct immunofluorescence of the perilesional skin showed a strong linear basement membrane zone (BMZ) band with c3 and a week BMZ IgG.(7,8) Patient was treated with methotrexate 15mg/week, tetracycline 500mgQID, Dapsone 100mgOD. Psoriatic lesions were improved after fifteen days, but she used to get occasional new blisters.Both conditions have subsided with post inflammatory hypopigmentation after one month. 


\section{CASE REPORT}

DISCUSSION: Since the initial description of coexistence of bullous disease and psoriasis by Bloom et al in 1929, several autoimmune bullous diseases associated with psoriasis have been reported in lecture. These include pemphigus vulgaris (PV),pemphigus foliaceous (PF), pemphigus herpetiformis, bullous pemphigoid (BP),linear bullous dermatosis (LAD), cicatricial pemphigoid (CP), epidermolysis bullosa acquisita(psoriasis bullosa acquisita) and a novel bullous dermatosis targeting 200kDa molecule present in the lower lamina lucida. $(9,10,7,8)$ Among these BP is the frequent condition to be associated with psoriasis (Psoriais pemphogoid), less than fifty cases of such association have been described in the English literature. This association is reported to be more common among men than women with an average age of onset at 63 years. In most cases, psoriasis preceded the development of BP, with an average stand interval of 20 yeas (Range, 1-60 years) between the two conditions.

The triggering factor in the association of psoriasis and BP remains unknown. Whether the treatment of psoriasis, pathological events at the basement zone (BMZ) in psoriasis itself, common immunological or immunogenic mechanisms, or a coincidence of multiple factors precipitates the autoimmune bullous diseases in these patients remain controversial. Since the coexistence was seen in patients who received a wide range of treatment for psoriasis as well as in untreated patients as well, it is difficult to incriminate a single agent as a potentially causative factor in the development of BP. Reduced barrier function of the diseased psoriatic epidermis, combined with the irritant effects of therapies administered for psoriasis, such as anthralin, tar and ultraviolet light, together with a lowgrade immunologic BMZ insult, may precipitate blister formation. Cram and Fukuyama demonstrated the triggering role of ultraviolet light $\mathrm{B}(\mathrm{UVB})$ in the induction of BP. Psoralen and UVA (PUVA) therapy is also known to induce blisters mimicking BP.(11) Recently, BP developing after efalizumab therapy for psoriasis has been described.(12) This molecule however, has been withdrawn now from the market due to serious neurological side effects.

Changes at the BMZ in psoriasis itself may be responsible for precipitating the bullous disease. The presence of chronic inflammation, trafficking of activated lymphocytes and abundance of antigen presenting cells in psoriasis could unmask, expose or alter BMZ antigens, giving rise to autoantibody production. Electron microscopy studies have shown a focal discontinuity and reduplication of the basal lamina in psoriasis, the concept of "epitope spreading" whereby tissue damage from a primary inflammatory process causes the release and exposure of a previously "sequestered" antigens. Leading to a secondary autoimmune response against the newly released antigen, may provide us with a unifying explanation for the development of subepidermal bullous disorders.

Common immunogenic or immunological mechanisms may also play a crucial role in this disease association. Psoriasis has been associated with other autoimmune diseases such as discoid and systemic lupus erythematosus, myasthenia gravis, chron's disease and ulcerative colitis. It may be suggested that psoriasis provides a particular predisposition of immune system that, under circumstances leads to auto immune response. Circulating antibodies against components of the malphigian layer and stratum corneum have been detected in the serum of psoriatic patients. The dysregulation of T-cell activity may result in the induction of specific antibodies to basement membrane antigens. Psoriasis has been historically considered as Th-1 type immune mediated disease. However, recent studies have established that Th-17 is the primary pathogenic subset,(13) this subset of T-cell plays a key role in autoimmunity.

Methotrexate has been used with good effect to treat BP associated psoriasis. Steroids should not be used for the fear of triggering pustular psoriasis. Other drugs that have shown to be beneficial 
include dapsone, azathioprine, cyclosporine, erythromycin with etretinate. We have treated our patient with methotrexate $15 \mathrm{mg}$ per week, tetracycline $500 \mathrm{mg}$ 6hourly.After one month both conditions responded well with resultant post inflammatory hyperpigmentation. Because of rarity in its association, we are reporting this case.

\section{REFERENCES:}

1. De Felice C, Chimenti S. Overview of Psoriasis. In: Chimenti S, editor. Psoriasis. Florence: SeeFirenze; 2005.

2. Saraceno R, Chimenti S. Associated conditions of psoriasis. In: Chimenti S, editor. Psoriasis. Florence: See- Firenze; 2005. p. 105-11.

3. Bloom D. Psoriasis with superimposed bullous eruption. Med J Rec 1929; 130: 246.

4. Grunwald MH, David M, Feuerman EJ. Coexistence of psoriasis vulgaris and bullous diseases. J Am Acad Dermatol 1985; 13: 224-8.

5. . Barnadas MA, Gilaberte M, Pujol R, Agustí M, Gelpí C, Alomar A. Bullous pemphigoid in a patient with psoriasis during the course of PUVA therapy: Study by ELISA test. Int J Dermatol 2006; 45: 1089-92.

6. Chen KR, Shimizu S, Miyakawa S, Ishiko A, Shimizu H, Hashimoto T. Coexistence of psoriasis and an unusual IgG-mediated subepidermal bullous dermatosis: Identifi cation of a novel 200kDa lower lamina lucida target antigen. Br J Dermatol 1996; 134: 340-6.

7. Wilczek A, Sticherling M. Concomitant psoriasis and bullous pemphigoid: coincidence or pathogenic relationship? Int J Dermatol 2006; 45: 1353-7.

8. Kirtschig G, Chow ET, Venning VA, Wojnarowska FT. Acquired subepidermal bullous diseases associated with psoriasis: A clinical, immunopathological and immunogenetic study. Br J Dermatol 1996; 135: 738-45.

9. Endo Y, Tamiira A, Ishikawa O, Miyachi Y, Hashimoto T. Psoriasis vulgaris coexistent with epidermolysis bullosa acquisita. Br J Dermatol 1997:137:785-6.

10. Morris SD, Mallipeddi R, Oyama N, Gratian MJ, Harman KE, Bhogal BS, et al. Psoriasis bullosa acquisita. Clin Exp Dermatol 2002; 27: 665-9.

11. Thomsen K, Schmidt H. PUVA-induced bullous pemphigoid. Br J Dermatol 1976; 95: 568-9.

12. Duong TA, Buffard V, André C, Ortonne N, Revuz J, Bagot M, et al. Efalizumab induced bullous pemphigoid. J Am Acad Dermatol 2010; 62: 161-2. Clin Exp Dermatol 2005; 30: 643-5.

13. Griffi ths CE, Barker JN. Psoriasis. In: Burns T, Breathnach S, Cox N, Griffi ths C, editors. Rook's Textbook of Dermatology. 8th ed. Oxford: Wiley Blackwell; 2010 .p. 20. 1-20. 60. 


\section{CASE REPORT}

Fig. 1: Multiple psoriatic plaques few erosions over lower back.

Fig. 2: Tense bullae filled with hemorrhagic fluid, erosions over thigh.

Fig. 3: Erosions and small scaly plaques over fore arm.

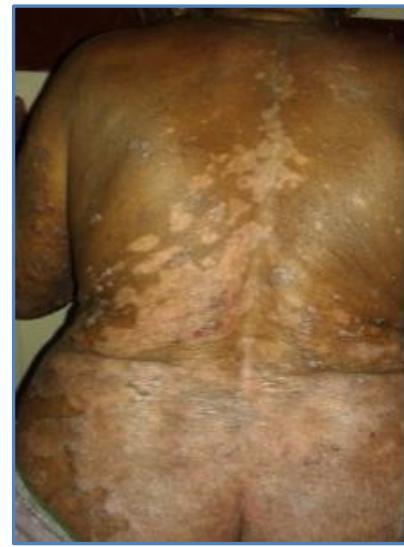

Fig. 1

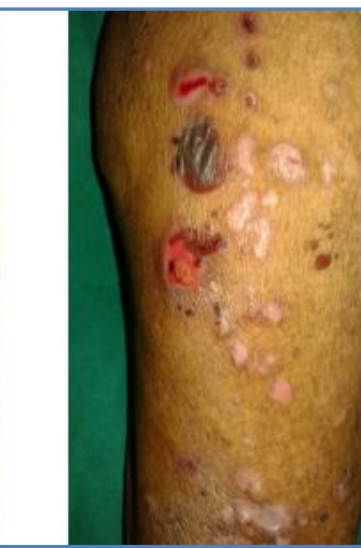

Fig. 2

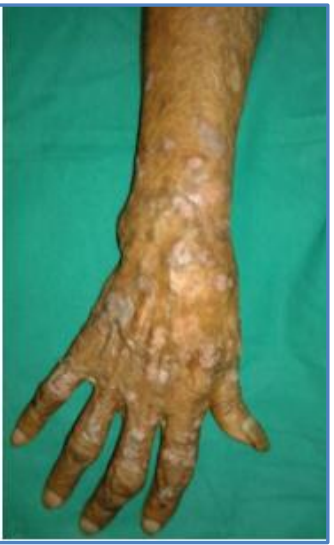

Fig. 3

Fig. 4: Hpe 10x-suprapapillary thinning, elongated club shaped rete ridges, collection of neutrophils in dermal papillae.

Fig. 5: Hpe40x-suprapapillary thinning, club shaped rete ridges, inflammatory cell infiltrate.

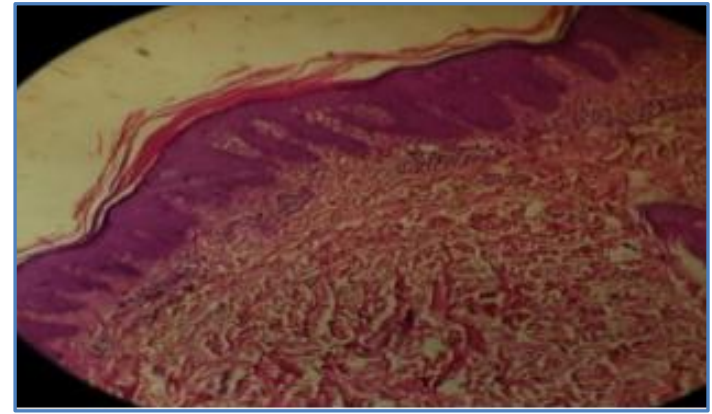

Fig. 4

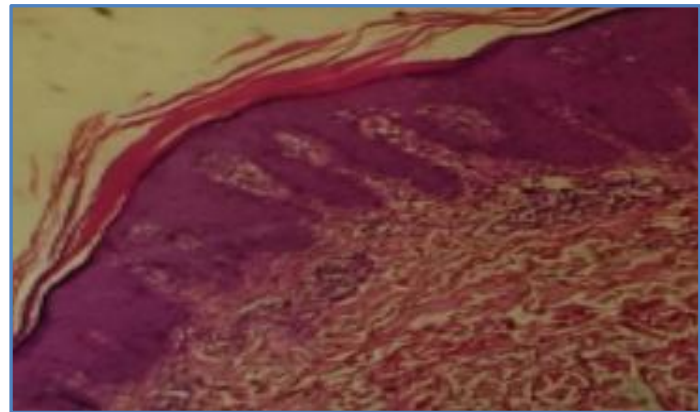

Fig. 5

Fig. 6: Hpe-10x-sub epidermal cleft with inflammatory infiltrate at roof and floor of blister.

Fig. 7: Hpe 40x- sub epidermal cleft with inflammatory cell infiltrate.

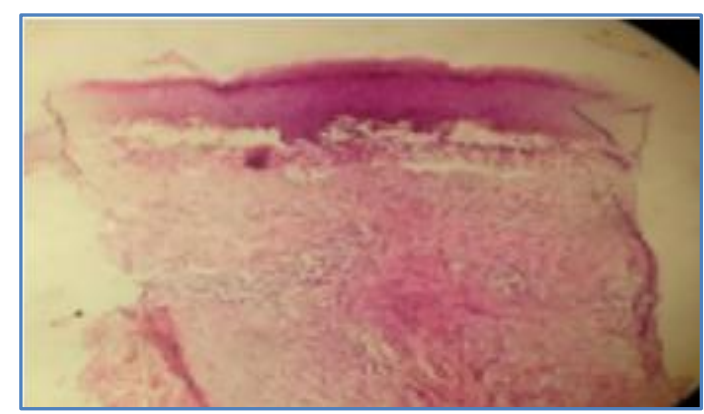

Fig. 6

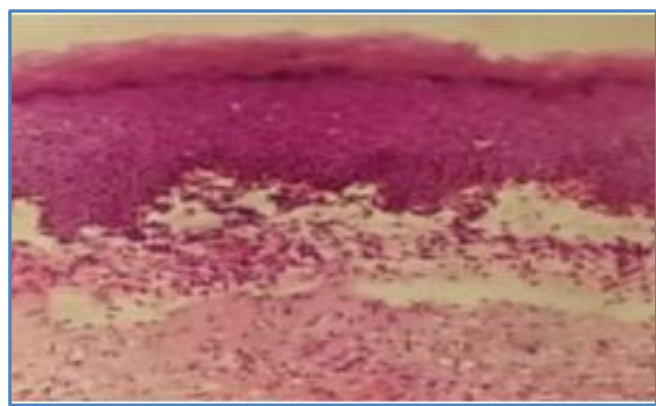

Fig. 7 


\section{CASE REPORT}

\section{AUTHORS:}

1. S. Dhanyasree

2. Ajaykumar

3. Chenna Subhashini

4. P. Anila Sunandhini

5. V.Srilakshmi

\section{PARTICULARS OF CONTRIBUTORS:}

1. Assistant Professor, Department of Dermatology, Andhra Medical College.

2. Assistant Professor, Department of Dermatology, Andhra Medical College.

3. Assistant Professor, Department of Dermatology, Andhra Medical College.

FINANCIAL OR OTHER

COMPETING INTERESTS: None
4. Associate Professor, Department of Dermatology, Andhra Medical College.

5. Junior Resident, Department of Dermatology, Andhra Medical College.

\section{NAME ADDRESS EMAIL ID OF THE CORRESPONDING AUTHOR:}

Dr. S. Dhanyasree Assistant Professor,

Department of Dermatology Veneriology

\& Leprosy, Andhra Medical College,

Visakhapatnam.

E-mail: drsrilakshmi99@gmail.com

Date of Submission: 10/08/2015.

Date of Peer Review: 11/08/2015.

Date of Acceptance: 24/08/2015.

Date of Publishing: 27/08/2015. 